\title{
Proof-of-Concept Validation of SDN-Controlled VCSEL- based S-BVTs in Flexi-Grid Optical Metro Networks
}

\author{
R. Martínez ${ }^{1}$, R. Casellas ${ }^{1}$, M. Svaluto Moreolo ${ }^{1}$, J. M. Fabrega ${ }^{1}$, R. Vilalta ${ }^{1}$, R. Muñoz ${ }^{1}$, L. Nadal ${ }^{1}$, J. P. \\ Fernández-Palacios ${ }^{2}$ \\ ${ }^{1}$ Centre Tecnòlogic de Telecomunicacions de Catalunya (CTTC/CERCA), Castelldefels (Barcelona), Spain \\ ${ }^{2}$ Telefónica I+D, GTCO, Don Ramón de la Cruz 82-84, Madrid, Spain \\ ricardo.martinez@cttc.es
}

\begin{abstract}
We model via YANG an adaptive, modular and programmable VCSEL-based S-BVT for optical flexi-grid metro networks. Discussion and experimental validation of the PCECC TSDN controller interfaces for automatic provisioning of flexi-grid connections are provided. OCIS codes: (060.4256) Networks, circuit-switched, (140.7260) Vertical cavity surface emitting lasers.
\end{abstract}

\section{Introduction}

Optical metro networks are rapidly evolving towards an extremely dynamic scenario, dealing with a huge increase of traffic characterized by high peaks. To support such bandwidth-consuming services and address stringent constraints on cost and power consumption within the metro network segment, a suitable choice of photonic enabling technologies is key as well as the use of flexible transport solutions (e.g., optical flexi-grid) controlled by software defined networking (SDN). Thus, the adoption of dense photonic integration and cost-effective photonic devices becomes crucial to implement network elements, like programmable adaptive transceivers characterized by reduced cost, power consumption and footprint. Vertical-cavity surface-emitting laser (VCSEL) technology at longwavelength (either tunable or large-bandwidth) arises as a potential candidate, which has been explored for the design of novel (SDN-enabled) sliceable bandwidth variable transceivers (S-BVTs) [1][2][3].

Within European PASSION project [4], a novel transceiver architecture based on large bandwidth VCSELs is envisioned to deal with the above metro network challenges. Particularly, a scalable SDN-controlled S-BVT able to generate multiple flows, suitably enabling/disabling a set of VCSELs operating at different wavelengths covering the entire $\mathrm{C}$-band, is proposed to achieve $\mathrm{Tb} / \mathrm{s}$ capacity with an integrated design. By exploiting adaptive multicarrier modulation, coherent $(\mathrm{CO})$ detection and multiple dimensions (spectral/spatial and polarization) the transceiver performance is enhanced in terms of flexibility, capacity and reach.

In this work, we formally model (via YANG) the PASSION S-BVT architecture as well as the required control interface (REST API) for its automatic SDN programmability. The adopted transport SDN (T-SDN) controller implements a Path Computation Element Central Controller (PCECC) [5] which dynamically processes flexi-grid variable data rate connections, computes the network resources (i.e., SBVT's devices, spatial and spectral path) and allocates them using both REST API for S-BVT configuration and PCE protocol (PCEP) for the optical switch programmability. An experimental validation of the T-SDN controller operations is conducted and presented.

\section{Adopted Flexi-Grid Optical Metro Infrastructure: VCSEL-based S-BVT Architecture}

Fig. 1(a) shows the proposed S-BVT architecture. Four SubModules, consisting of 10 VCSELs each, are integrated on a silicon-on-insulator ( $\mathrm{SOI}$ ) chip to achieve a compact transmitter ( $\mathrm{Tx}$ ) Module with $2 \mathrm{~Tb} / \mathrm{s}$ maximum capacity. It is assumed that each $20 \mathrm{GHz}$-bandwidth VCSEL is directly modulated with adaptive multicarrier modulation formats (either DMT or OFDM) giving up to $50 \mathrm{~Gb} / \mathrm{s}$. The VCSEL can be enabled/disabled and its operating wavelength fine-tuned, in a range of $75 \mathrm{GHz}$ with respect to the central frequency $(f c)$ according to the T-SDN controller decisions. This allows to suitably adapt the generated flow to the adjacent sub-channels, forming a multi-flow superchannel according to the network requirements, and eventually mitigating the spectrum fragmentation.

The channel spacing depends on the number of VCSELs in the Module (covering the entire C-band), as shown in Fig. 2(b). Sub-wavelength granularity and variable bandwidths (per flow) are enabled by adaptive electrical/digital multicarrier modulation. The S-BVT can be upgraded to achieve higher capacity by adding SOI-chip fundamental Modules, up to $8 \mathrm{~Tb} / \mathrm{s}$ for a fully-equipped (single polarization) architecture, populating the entire C-band with $25 \mathrm{GHz}$-spaced channels (at $50 \mathrm{~Gb} / \mathrm{s}$ per each VCSEL flow). At the receiver (Rx) side, the S-BVT is equipped with an arbitrary set (to grow-as-needed) of integrated CO-Rxs with tunable local oscillators, operating at the suitable central frequencies to correctly recover the transmitted flows. The S-BVT is adaptive to multiple rate/reach according to the traffic demand and selected path, for a target performance related to FEC choice.

In the targeted S-BVT, 40 VCSELs (in 4 Modules and 4 SubModules) are equipped occupying the optical spectrum between 191.000-195.000THz. Assigned $f c$ per VCSEL is associated to a given Module Id and SubModule 
Id. As shown in Fig. 1(b), a VCSEL's $f_{c}$ is determined by the tuple Module Id / SubModule Id / VCSEL Id (e.g., $f_{c}$ at $191.000 \mathrm{THz}$ is assigned to VCSEL-1 on SubModule 1 within Module 1). Fig. 1(c) describes the S-BVT's YANG data model for both Tx and Rx. For a given Module Id and Submodule Id, the defined VCSEL's attributes are: Id, used-state, optical bandwidth, central frequency (i.e., $f c)$, supported frequency tunability $( \pm 75 \mathrm{GHz})$, selected operating central frequency, FEC, modulation format, baud and bit rates (up to $50 \mathrm{~Gb} / \mathrm{s}$ ). On the other hand, each receiver in a given Module is determined by its Id, used-state and tunable local oscillator (i.e., central frequency).

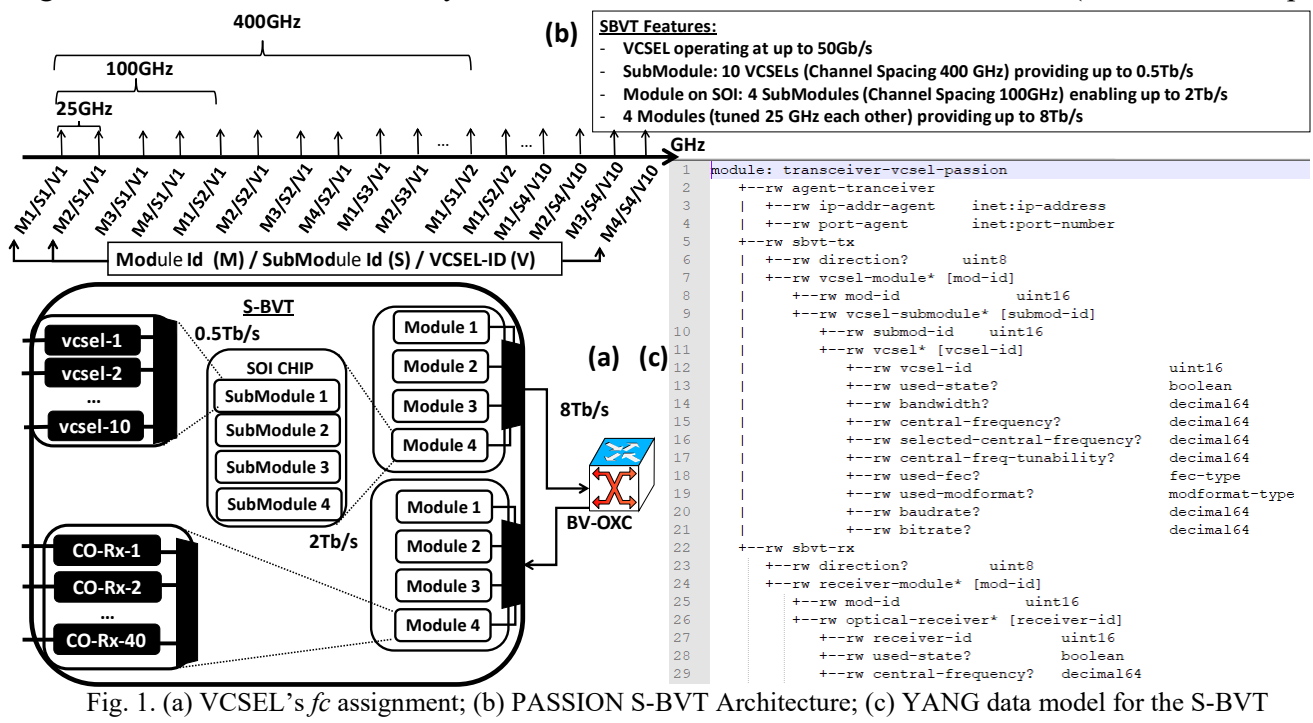

3. SDN-Controller: Architecture, Control Interfaces and Workflows

Fig. 1. (a) depicts the implemented PCECC-based T-SDN controller for the automatic computation and programmability of optical flexi-grid connections. The topology manager gathers network and resource information stored in the TE Database (TED) containing the node connectivity and link attributes (e.g., optical spectrum availability, link distance, etc.), updated via BGP-LS protocol, as well as the S-BVT status information (i.e., pool of VCSELs and CO-Rxs) attached to the BV-OXCs. The S-BVT information is collected via a REST API (GetSBVTStatusReq / GetSBVTStatusRsp messages) between the T-SDN controller and the S-BVT agents. Using TED information, upon receiving a connection demand (endpoints and data rate $-r$ - in $\mathrm{Gb} / \mathrm{s}$ ) the provisioning manager computes a spatial path (i.e., nodes and links), selects the S-BVT capabilities (i.e., set of VCSELs and CORxs) as well as the end-to-end optical frequency slot (FS). To this end, a Routing and Spectrum Assignment (RSA) algorithm computes the shortest path (in terms of distance) satisfying both the spectrum continuity and contiguity constraints [5]. Specifically, the RSA first determines the number of VCSELs $\left(n_{V C S E L}\right)$ to satisfy $r$ by $n_{V C S E L}=$ upper integer of $r / r_{V C S E L}$, where $r_{V C S E L}$ is the VCSEL's data rate (in [4], 50Gb/s). Notice that $n_{V C S E L}$ also determines the number of required CO-Rxs at the egress S-BVT. Next, the RSA seeks for a set of available and spectrally contiguous $n_{V C S E L}$ VCSELs. This in turn does constrain the end-to-end FS to be occupied in all path links. Fig. 2.b shows an example of a super-channel flexi-grid flow $(r=250 \mathrm{~Gb} / \mathrm{s})$ requiring 5 spectrum-contiguous VCSELs.

The output of the RSA algorithm is then passed to the provisioning manager to configure both ingress and egress S-BVTs and the involved path's BV-OXCs. For the S-BVT configuration, this is done by REST API ConfSBVTReq and ConfSBVTRsp messages. For the BV-OXCs configuration, PCECC PCEP messages are used [6]. The provisioning mechanism (workflow) for a flexi-grid connection is detailed Fig. 2(c) entailing three steps: 1) request processing (PCEP PCInitiate message), TED updating with S-BVTs' status (REST API), and RSA execution; 2) configuration of S-BVT's VCSELs' and CO-Rxs (REST API); 3) BV-OXCs configuration (PCECC PCEP messages). The successful establishment is then reported (PCEP PCRpt) message.

\section{Proof-of-Concept Validation}

Fig. 3 depicts the captured control messages exchanged between the T-SDN controller and S-BVT / BV-OXC agents to set up a new flexi-grid optical connection. Upon receiving the PCEP PCInitiate message from the demanding Application, the T-SDN controller retrieves the current status of all the S-BVTs via the REST GET method messages (GetSBVTReq/Rsp). The response carries S-BVT's VCSELs and Co-Rxs attributes are as discussed in the above YANG data model encoded using JSON notation. The RSA output entails the configuration of both selected ingress set of VCSELs and egress CO-Rxs using the REST POST method messages 
(ConfSBVTReq/Rsp). Next, the computed end-to-end FS in all the traversed path's BV-OXCs is programmed by the T-SDN controller relying on the PCECC's PCLabelUpd messages (PCEP Message 30) as described in [5]. Finally, the successful setting up of the optical flow request is reported to the On-Demand Bandwidth Application.

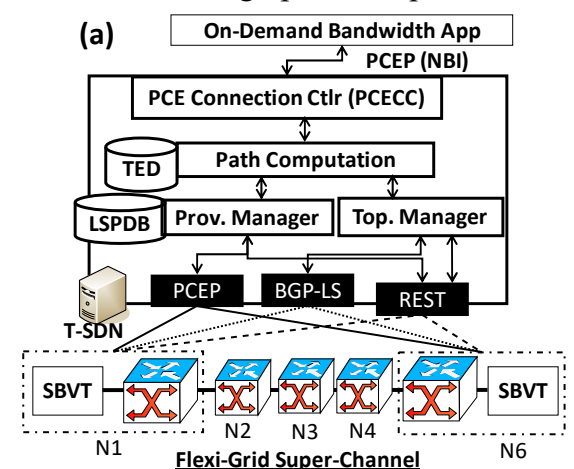

(b) FreqCentral: $192.025 \mathrm{GHz}$

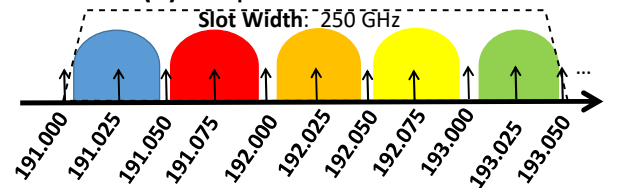

Fig. 2. (a) T-SDN controller architecture; (b) Optical flexi-grid Super Channel example; (c) Workflow flexi-grid connection set up

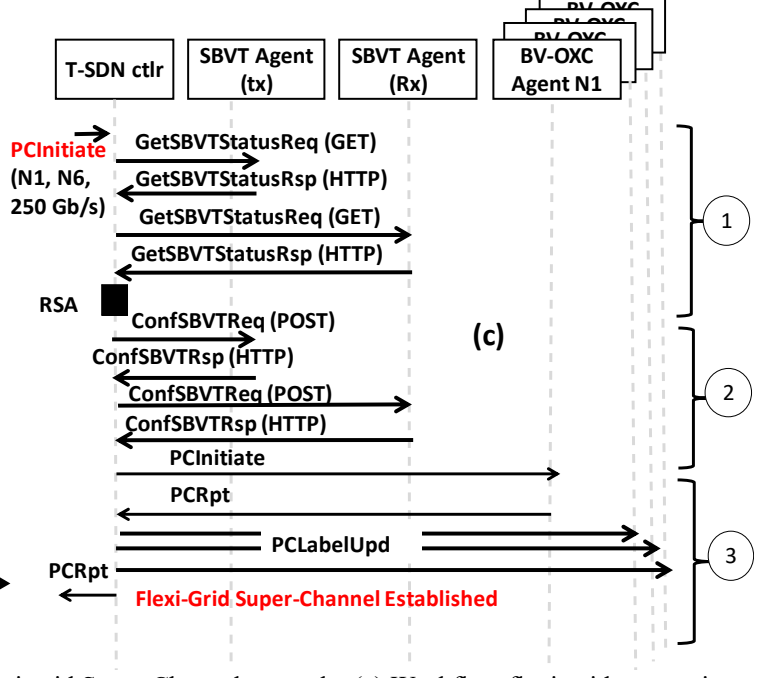

\section{Conclusions}

This work presents the YANG data model detailing the VCSELs and CO-Rxs devices of an adaptive, modular and programmable S-BVT designed in the European PASSION project for optical metro networks. The REST API control for retrieving S-BVTs information and the automatic configuration steered by a PCECC T-SDN controller when provisioning variable data-rate flexi-grid connections are thoroughly discussed and experimentally validated.

Acknowledgments. This work has been partially supported by the EU PASSION Project (780326) and the Spanish DESTELLO TEC201569256-R and AURORAS RTI2018-099178-B-I00 projects.

\begin{tabular}{|c|c|c|c|c|c|}
\hline 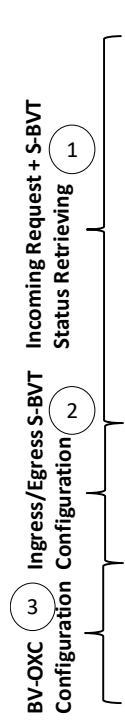 & $\begin{array}{l}\text { Source } \\
\text { On-Demand Bw App } \\
\text { T-SDN Ctlr } \\
\text { S-BVT/BV-OXC N1 } \\
\text { T-SDN Ctlr } \\
\text { S-BVT/BV-OXC N2 } \\
\text { T-SDN Ctlr } \\
\text { S-BVT/B-OXC N3 } \\
\text { T-SDN Ctlr } \\
\text { S-BVT/BV-OXC N4 } \\
\text { T-SDN Ctlr } \\
\text { S-BVT/BV-OXC N5 } \\
\text { T-SDN Ctlr } \\
\text { S-BVT/BV-OXC N6 } \\
\text { T-SDN Ctlr } \\
\text { S-BVT/BV-OXC N7 } \\
\text { T-SDN Ctlr } \\
\text { S-BVT/BV-OXC N8 } \\
\text { T-SDN Ctlr } \\
\text { T-SDN Ctlr } \\
\text { S-BVT/BV-OXC N1 } \\
\text { S-BVT/BV-OXC N1 } \\
\text { T-SDN Ctlr } \\
\text { S-BVT/BV-OXC N6 } \\
\text { T-SDN Ctlr } \\
\text { T-SDN Ctlr } \\
\text { T-SDN Ctlr } \\
\text { T-SDN Ctlr } \\
\text { T-SDN Ctlr } \\
\text { T-SDN Ctlr }\end{array}$ & $\begin{array}{l}\text { Destination } \\
\text { T-SDN Ctlr } \\
\text { S-BVT/BV-OXC N1 } \\
\text { T-SDN Ctlr } \\
\text { S-BVT/BV-OXC N2 } \\
\text { T-SDN Ctlr } \\
\text { S-BVT/B-OXC N3 } \\
\text { T-SDN Ctlr } \\
\text { S-BVT/BV-OXC N4 } \\
\text { T-SDN Ctlr } \\
\text { S-BVT/BV-OXC N5 } \\
\text { T-SDN Ctlr } \\
\text { S-BVT/BV-OXC N6 } \\
\text { T-SDN Ctlr } \\
\text { S-BVT/BV-OXC N7 } \\
\text { T-SDN Ctlr } \\
\text { S-BVT/BV-OXC N8 } \\
\text { T-SDN Ctlr } \\
\text { S-BVT/BV-OXC N1 } \\
\text { S-BVT/BV-OXC N1 } \\
\text { T-SDN Ctlr } \\
\text { T-SDN Ctlr } \\
\text { S-BVT/BV-OXC N6 } \\
\text { T-SDN Ctlr } \\
\text { S-BVT/BV-OXC N4 } \\
\text { S-BVT/B-OXC N3 } \\
\text { S-BVT/BV-OXC N2 } \\
\text { S-BVT/BV-OXC N6 } \\
\text { S-BVT/BV-OXC N1 } \\
\text { On-Demand Bw ADp }\end{array}$ & $\begin{array}{l}\text { Protocol } \\
\text { PCEP } \\
\text { HTTP } \\
\text { HTTP } \\
\text { HTTP } \\
\text { HTTP } \\
\text { HTTP } \\
\text { HTTP } \\
\text { HTTP } \\
\text { HTTP } \\
\text { HTTP } \\
\text { HTTP } \\
\text { HTTP } \\
\text { HTTP } \\
\text { HTTP } \\
\text { HTTP } \\
\text { HTTP } \\
\text { HTTP } \\
\text { PCEP } \\
\text { HTTP } \\
\text { HTTP } \\
\text { PCEP } \\
\text { HTTP } \\
\text { HTTP } \\
\text { PCEP } \\
\text { PCEP } \\
\text { PCEP } \\
\text { PCEP } \\
\text { PCEP } \\
\text { PCEP }\end{array}$ & 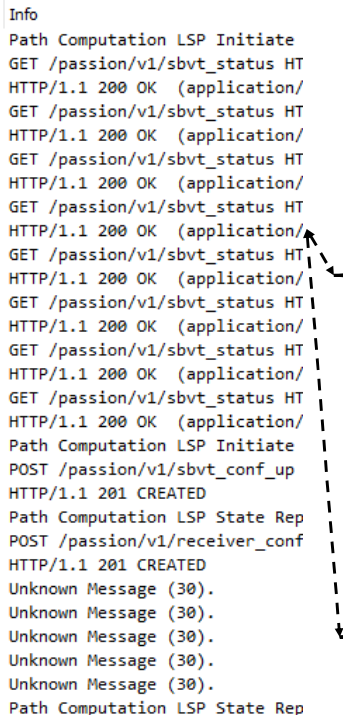 & 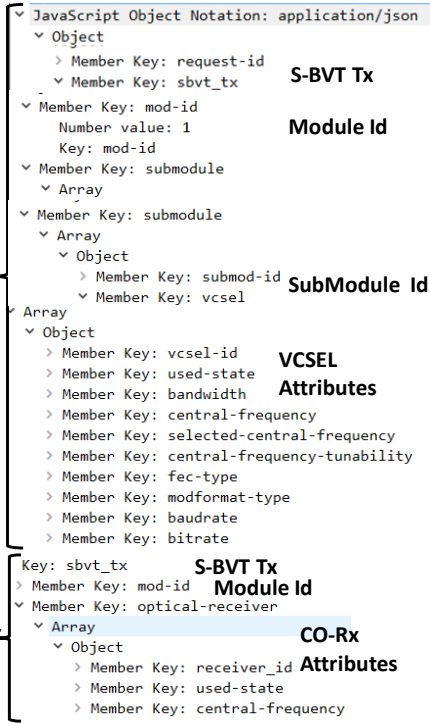 \\
\hline
\end{tabular}

Fig. 3. T-SDN control messages (and JSON Encoding) for programming both VCSEL-based S-BVT and BV-OXCs.

\section{References}

[1] M. Svaluto Moreolo, et al., "Modular SDN-enabled S-BVT Adopting Widely Tunable MEMs VCSEL for Flexible/Elastic Optical Metro Networks", in Proc. of OFC 2018, M1A.7, March 2018.

[2] M. Svaluto Moreolo, et al., "Exploring the Potential of VCSEL Technology for Agile and High Capacity Optical Metro Networks" ONDM'18

[3] A. Malacarne, et al., "Software-Defined Reconfigurable VCSEL-based Transmission", Optics Express, 26 (7), April 2018.

[4] EC H2020 Photonic KET PASSION Project, http://www.passion-project.eu

[5] R. Martínez, et. al., "Distributed vs. Centralized PCE-based Transport SDN Controller for Flexi-Grid Optical Networks", OFC 2017 